\title{
Test of the absolute effect of size on heaviness
}

\author{
SERGIO CESARE MASIN \\ University of Udine, Udine, Italy \\ and \\ LOREDANA CRESTONI and VALENTINA FANTON \\ University of Padua, Padua, Italy
}

The size-weight illusion shows up when the heaviness of objects of different sizes and equal weights is evaluated either by comparing directly two objects or by rating one object at a time. The rating method also implies a comparison: The object seen is compared indirectly with other remembered objects from the size $x$ weight factorial design by subjects who rate the heaviness of all objects defined by the design. It is possible, therefore, that the illusion depends on the relative size, rather than on the absolute size, of the compared objects. The experiment reported here tests this possibility by using different groups of subjects for each of the sizes used. The results show that the size-weight illusion depends on the absolute size of objects.

The size-weight illusion (SWI) occurs when two objects of different sizes and equal weights are compared. The smaller of two objects compared simultaneously is usually reported to be heavier than the other (Charpentier, 1891). The SWI also occurs when the heaviness of single objects is rated. Small objects are rated heavier than large ones of equal weight (Anderson, 1970).

Given three objects of equal weight with sizes S1, S2, and $\mathrm{S} 3$ in the relation $\mathrm{S} 1>\mathrm{S} 2>\mathrm{S} 3$, the object of size S2 feels heavier when compared with the object of size $S 1$ and lighter when compared with the object of size S3. Thus it is possible that the SWI depends on the size relation between the compared objects, rather than on the absolute sizes of the objects.

It could be thought that the results of the rating method, which requires an evaluation of the heaviness of one object at a time, prove that there is an effect of absolute size on heaviness. However, in experiments on the SWI, each single object whose heaviness is rated is one of a set of objects of different sizes that are lifted by the subject, so it is possible that the illusion results from a comparison of the object with memories of the previously lifted objects. A proper test of the absolute effect of size on heaviness can be obtained only by showing subjects objects of one size and by using a different group of subjects for each different size. The experiment reported here was aimed at obtaining a test of the SWI by using this procedure.

\section{METHOD}

\section{Subjects}

The subjects were 96 university students recruited upon entering the psychology department at the University of Padua.

Mail should be directed to S. C. Masin at the University of Udine, Institute of Philosophy, Pedagogics, and Teaching of Modern Languages, Via Antonini 8, 33100 Udine, Italy.

\section{Stimuli}

Weights. The stimuli for heaviness were gray cylindrical plastic botthes that were $4.5 \times 7,7.5 \times 13$, or $9 \times 17 \mathrm{~cm}$ in width and height, respectively. A $3 \times 5$ (size $\times$ weight) factorial design was used. The five levels of weight were $150,225,340,510$, and $765 \mathrm{~g}$. The weights were varied by filling the interiors of the bottles with lead shot and cotton. A string with a ring was tied to the top of each bottle for lifting. Two standards were used, one of $100 \mathrm{~g}$ and one of $1,150 \mathrm{~g}$.

Reflectances. The stimuli for lightness were five $2.2 \times 2.2 \mathrm{~cm}$ squares stuck in the middle of a $20 \times 20 \mathrm{~cm}$ square with reflectance of .31 . The reflectance of a stimulus was $.11, .19, .40, .52$, or .67 . Two standards were used, with reflectances of .04 and .87 .

\section{Apparatus}

The apparatus for weight lifting was the same as that used by Masin and Crestoni (in press). The subject stood in front of a screen in correspondence with a mark. The experimenter, on the other side of the screen, placed the string of a bottle in two pulleys on each side of the top of the screen. The subject lifted the bottle by pulling down the ring hanging on his/her side of the screen. A sliding panel with an opening served to show, or to hide, the bottle.

The squares were shown through the opening in the sliding panel, and were placed on the frontal parallel plane. The subject viewed the squares from a distance of $2 \mathrm{~m}$ while sitting on a chair.

\section{Procedure}

Three groups of 32 subjects participated in three sessions (one group for the small, one for the medium, and one for the large bottles). In the first session, all groups rated once the lightness of the squares. In the second session, each group lifted twice consecutively the five bottles of one size. In the third session, all groups again rated the lightness of the squares. Both the bottles and the squares were presented in random order. The standards for heaviness were presented before every two bottles. Also, the standards for lightness were presented before every two squares (to make this possible, half the subjects rated six squares in the first session and four squares in the third; the other half rated four squares in the first session and six in the third). Half the subjects were first shown the heavy and black standards, and the other half the light and white standards.

The ratings were made using the integers $1-20$. For heaviness, 1 was defined to correspond to the light standard and 20 to the heavy standard. To secure the same reference points for all three groups, the standards for weights never were made visible. For reflectance, 1 was defined to correspond to the black standard and 20 to the white standard. Reflectance was scaled to check whether or not the three different groups of subjects used numbers in the same fashion. 

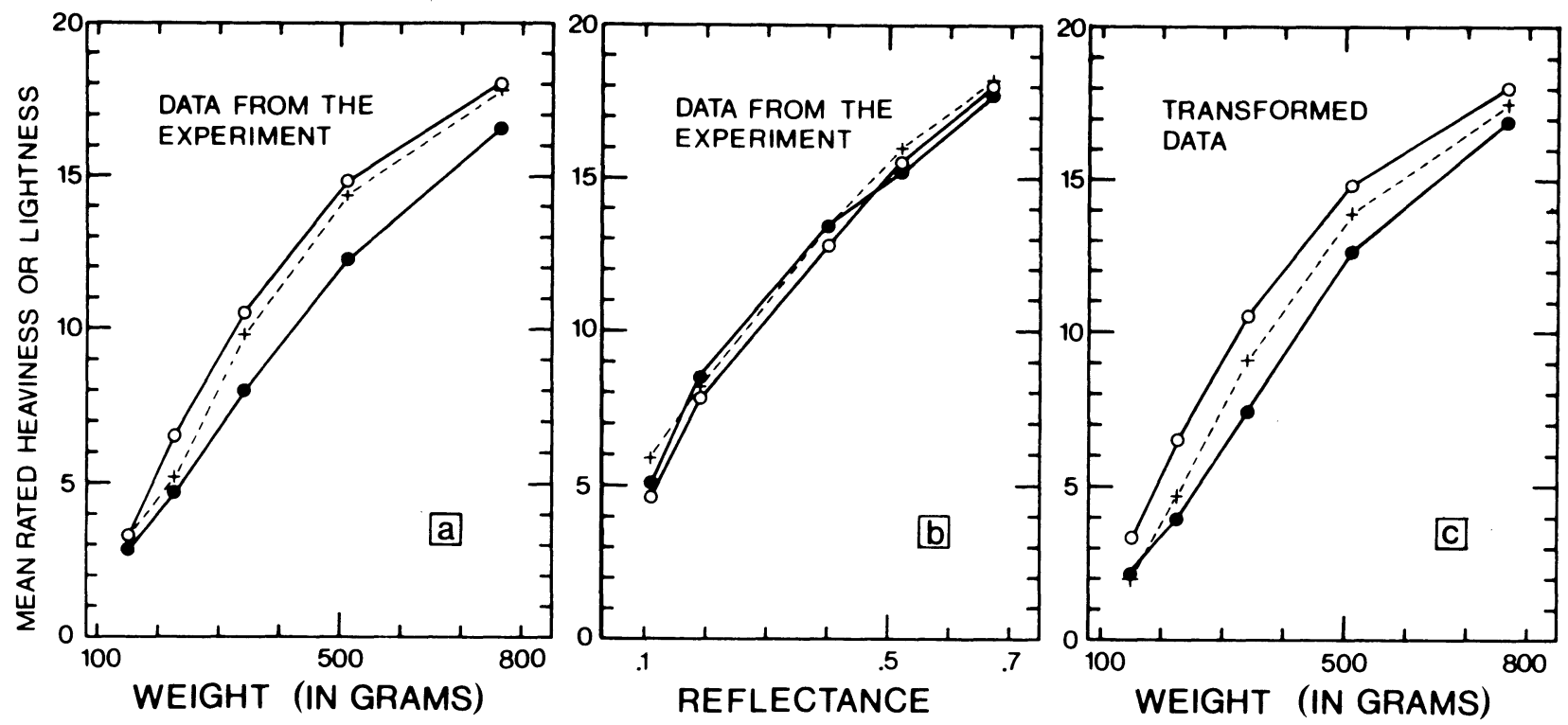

Figure 1. Psychophysical functions relating (a) mean rated heaviness to weight in grams and (b) mean rated lightness to reflectance. The empty dots, pluses, and filled dots represent the results for the groups that lifted only small, medium, or large bottles, respectively. Also represented is (c) the mean rated heaviness as a function of weight when the relative effect due to the use of numbers is eliminated.

Each bottle was placed behind the sliding panel after the panel was moved all the way to the subject's right. The sliding panel was then moved all the way to the left to show the bottle. The subject lifted the bottle while seeing it, and rated its heaviness in reference to the heaviness of the remembered unseen standards. After each rating, the sliding panel was moved all the way to the right.

\section{RESULTS AND DISCUSSION}

The results are depicted in Figure 1. In Figure 1a, the mean rated heaviness is reported on the ordinate and the weight in grams on the abscissa. The empty dots, the pluses, and the filled dots represent the mean results for the small, medium, and large bottles, respectively.

In Figure 1b, the mean rated lightness is represented on the ordinate and the reflectance of squares on the $a b-$ scissa. The empty dots, the pluses, and the filled dots represent the results from the groups of subjects who rated the heaviness of small, medium, and large bottles, respectively. It may be seen that the three groups differed slightly in the use of numbers.

The relative effect due to the different use of numbers can be eliminated in Figure 1a by subtracting algebraically the distance between the means represented by pluses and filled dots and the corresponding means represented by empty dots in Figure $1 \mathrm{~b}$ from the respective means represented by pluses and filled dots in Figure 1a.
Figure 1c represents the results for the SWI when the relative effect of the use of numbers is so eliminated.

Each graph in Figure 1c was obtained from a different group of 32 subjects. Masin and Crestoni (in press, Experiment 2) obtained essentially the same graphs from a single group of 20 subjects who rated the heaviness of the same bottles in the same stimulus conditions as in this experiment. Since, in Masin and Crestoni's experiment, the SWI was shown to occur with high statistical significance by an analysis of variance, a statistical test is superfluous for the results of the present experiment. It may be concluded, therefore, that this experiment shows that the SWI occurs, and that the SWI does not depend on the relative size of bottles, but rather on the absolute size of the lifted objects.

\section{REFERENCES}

Anderson, N. H. (1970). Averaging model applied to the size-weight illusion. Perception \& Psychophysics, 8, $1-4$.

Charpentier, A. (1891). Analyse experimentale de quelques elements de la sensation de poids. Archives de Physiologie Normal et Pathologique, 3, 122-135.

Masin, S. C., \& CRestoni, L. (in press). Experimental demonstration of the sensory basis of the size-weight illusion. Perception \& Psychophysics.

(Manuscript received for publication January 25, 1988.) 